\title{
Use of Epiphysiodesis as Treatment for a Proximal Physeal Tibial Fracture in a Dog
}

\author{
Pierre P. Picavet ${ }^{1} \quad$ Bernard Bouvy $^{1} \quad$ Martin Hamon $^{1} \quad$ Michael Lefebvre $^{1} \quad$ Marc Balligand $^{1}$ \\ ${ }^{1}$ Department of Clinical Sciences, FARAH, Faculty of Veterinary \\ Medicine, University of Liège, Liège, Belgium \\ Address for correspondence Pierre P. Picavet, DVM, Department of \\ Clinical Sciences, FARAH, Faculty of Veterinary Medicine, University of \\ Liège, Quartier Vallée 2, Avenue de Cureghem 3-B44, Liège, Belgium \\ VCOT Open 2019;2:e55-e59. \\ (e-mail: pierre.picavet@uliege.be).
}

\begin{abstract}
Keywords

- tibial plateau slope

- epiphysiodesis

- growing dogs

- Salter-Harris II fracture

Objective The aim of this study was to describe the use of epiphysiodesis by the means of a screw to treat a proximal tibial Salter-Harris II fracture associated with a tibial tuberosity avulsion in a 4.5-month-old Airedale Terrier.

Study design A healing proximal tibial epiphyseal fracture was observed after a 10day period. Tibial plateau angle was $40^{\circ}$. The fracture was treated by the insertion of a fluoroscopy-guided 3.5-mm cancellous screw.

Results Successful healing of fractures and levelling of tibia plateau were obtained. Final tibial plateau angle was $8^{\circ}$. At long-term follow-up (18 months), owners reported sustained and full functional recovery.

Conclusion Prior to ossification of the proximal tibial physis, epiphysiodesis with a screw can be used as a treatment of sub-acute, moderately displaced, Salter-Harris I or II fractures.
\end{abstract}

\section{Introduction}

Fractures of the proximal tibia are identified in about $7 \%$ of all canine tibial fractures. ${ }^{1-4}$ Avulsion of the tibial tuberosity and Salter-Harris type I or II fractures of the proximal physis are the most reported in immature dogs. ${ }^{1-3}$ Avulsion of the tibial tuberosity may occur either independently or in association with fracture-separation of the tibial epiphysis. Incidence reported in the literature varies from rare to common. ${ }^{5-8}$ Small breed dogs, particularly terriers, seem predisposed to the latter. ${ }^{5,7-10}$ This association of fractures has been reported in dogs aged between 3.5 and 8 months. ${ }^{7,9,10}$ The typical distocaudal displacement of the proximal segment is of concern as it is accompanied by subsequent increased tibial plateau angle. This predisposes to increased stress on the cranial cruciate ligament, possibly leading to its rupture. ${ }^{11}$

Currently, there are several ways to manage proximal tibial epiphyseal fractures in puppies. Kirschner wires with or without tension band are mostly used, ${ }^{7,8}$ reduction and fixation being performed either by open technique or percutaneously. ${ }^{12,13}$

received

January 16, 2019

accepted after revision

April 25, 2019
Proximal tibial epiphysiodesis has been described as a therapy for growing dogs with cranial cruciate ligament deficient stifles. It aims at closing the cranial part of the proximal tibial physis with a cancellous screw. ${ }^{14,15}$ This case report describes a hitherto unreported use of proximal tibial epiphysiodesis as mini-invasive treatment of a displaced Salter-Harris II proximal tibial fracture, associated with a tibial tuberosity avulsion, in a puppy.

\section{Clinical Report}

A 13.8-kg, 4.5-month-old, female, Airedale Terrier was presented with a right hindlimb lameness after trauma while playing with another dog, 10 days earlier. Faced with no improvement after conservative management, the dog was referred at the 'Clinique Vétérinaire Universitaire of Liège University'. General physical examination and neurological assessment were within normal limits. A numerical lameness scale $(0-5)$, as previously described, was utilized. ${ }^{16}$ Orthopaedic examination revealed a severe weight-bearing lameness (4/5). In comparison with the contralateral thigh, low-grade amyotrophy of the right thigh was present. Deep

\footnotetext{
(c) 2019 Georg Thieme Verlag KG Stuttgart · New York
}

License terms

10.1055/s-0039-1692170. ISSN $2625-2325$.

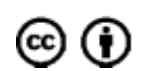


static pressure caused pain at the level of the tibial crest, but no crepitation could be elicited upon stifle mobilization. Direct and indirect drawer signs were negative. Patella was in normal position and stable within the trochlea. Radiographs of the affected stifle joint revealed soft tissue swelling cranial to the right tibial tuberosity and a Salter-Harris type II fracture of the right tibial proximal epiphysis associated with tibial tuberosity avulsion. The tibial plateau and tibial tuberosity were displaced as a single block, with the first being caudodistally tilted and the latest cranioproximally elevated with the presence of several mineralized nuclei distal to it ( $\mathbf{\text { Fig. }}$ 1). The tibial plateau angle was $40^{\circ}$. It was measured on the mediolateral view according to the method described by Slocum. ${ }^{17}$

Sedation was obtained with methadone $(0.2 \mathrm{mg} / \mathrm{kg}$, intravenous [IV]) and midazolam ( $0.2 \mathrm{mg} / \mathrm{kg}$, IV). Anaesthesia was induced with propofol (2-6 mg/kg, IV) given to effect and maintained with isoflurane. A peridural locoregional analgesia was performed (morphine $0.2 \mathrm{mg} / \mathrm{kg}$ and levobupivacaine $1 \mathrm{mg} / \mathrm{kg}$ ).

The surgical procedure was based on the one described by Vezzoni and colleagues. ${ }^{14}$ The lateral skin incision extended from slightly distally to the patella to the tibial tuberosity. The patellar ligament was reclined medially to allow, under a fluoroscopic guidance, the intra-articular insertion of a $32 \mathrm{~mm} \times 3.5 \mathrm{~mm}$ cancellous screw in a positional mode. The desired point of insertion was the mediolateral centre of the most proximal part of the tibial plateau. The joint was lavaged with saline. The surgical wound was routinely closed. Postoperative radiographs confirmed appropriate placement of the screw. Immediate postoperative tibial plateau angle was $36^{\circ}$ (-Fig. 2).

Cephalexin was administered $20 \mathrm{mg} / \mathrm{kg}$ perioperatively, methadone $0.2 \mathrm{mg} / \mathrm{kg}$ every 4 hours 24 hours postoperatively and carprofen $2 \mathrm{mg} / \mathrm{kg}$ twice a day for 5 days. Cold

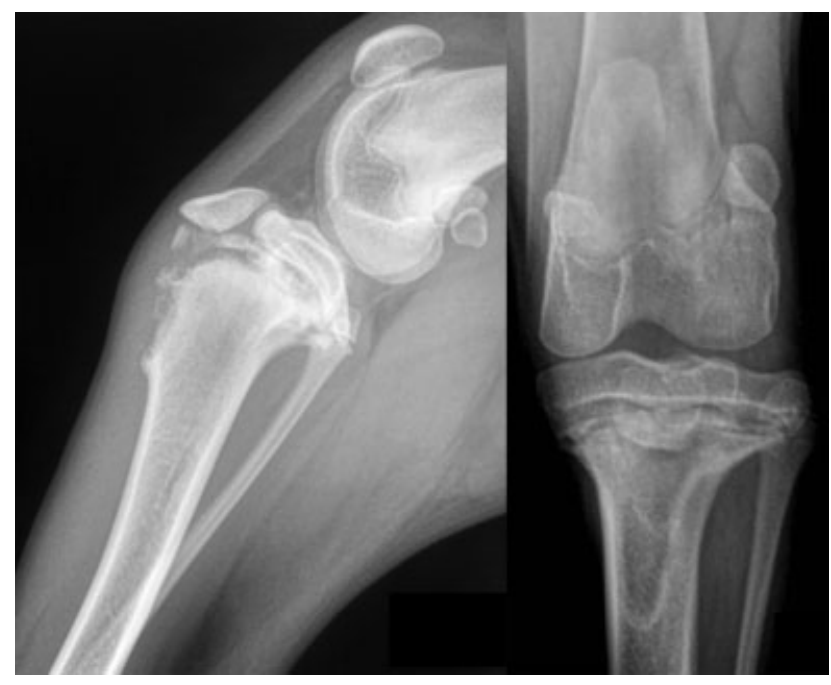

Fig. 1 Preoperative radiographs showing a moderately displaced Salter-Harris II fracture of the right tibial proximal epiphysis associated with tibial tuberosity avulsion.

packs were applied on the stifle four to six times a day for 5 minutes for 4 days. The dog was discharged from the hospital the day after surgery with a 5-day carprofen 2 $\mathrm{mg} / \mathrm{kg}$ twice a day and 3-day tramadol $2 \mathrm{mg} / \mathrm{kg}$ twice a day prescription. Strict exercise restriction was recommended until re-evaluation.

At recheck 3 weeks postoperatively, owners mentioned a decrease in the lameness at home. The dog still presented a stiff gait and a mild right hindlimb lameness (2/5) but no stifle pain. Direct and indirect drawer signs remained negative. Radiographs revealed patellar ligament thickening and joint swelling. Ongoing bone healing of tibial tuberosity fracture was present. Tibial plateau angle was $32^{\circ}$. Leash walking and passive mobilization of the right stifle were advocated.

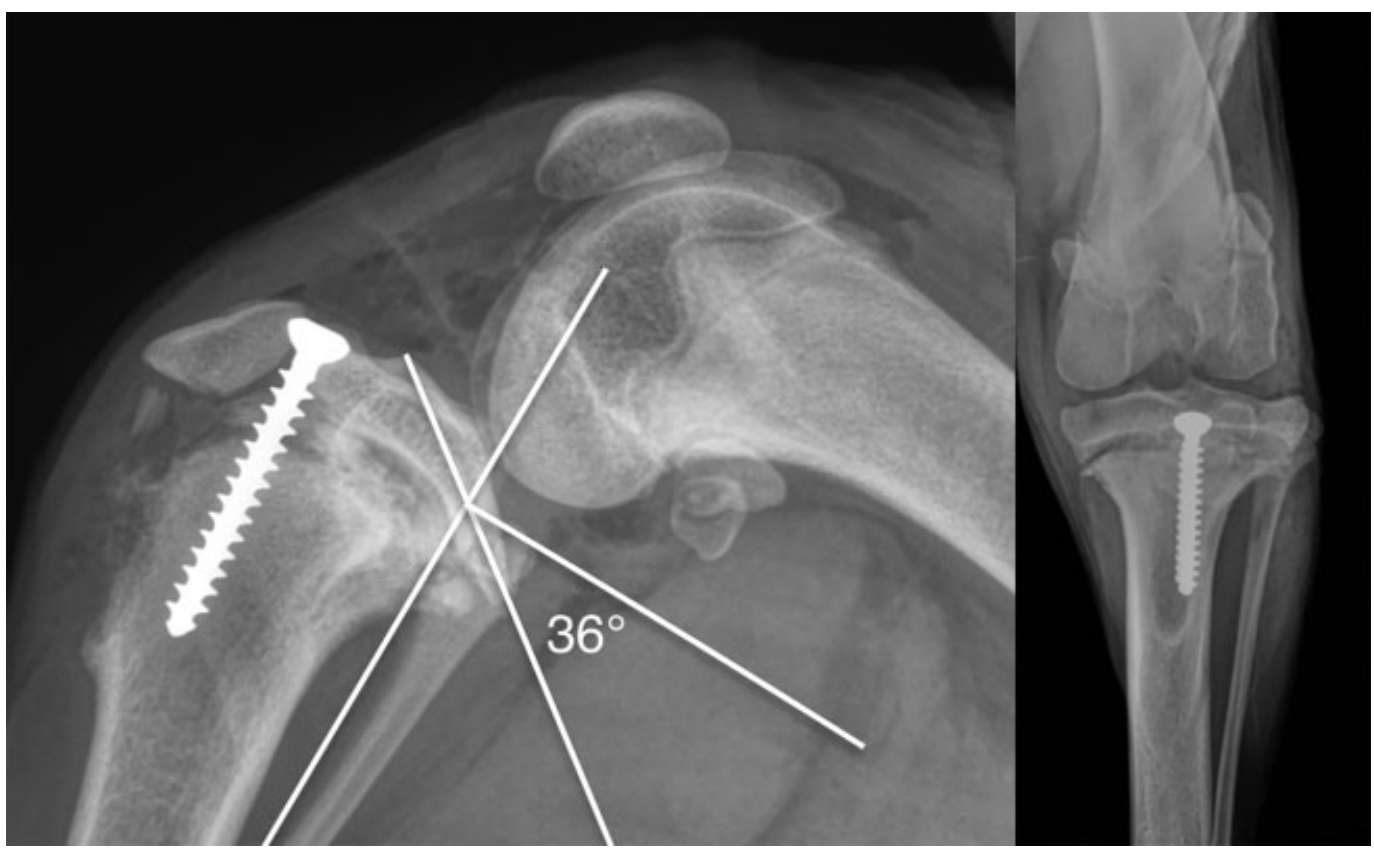

Fig. 2 Immediate postoperative radiographs showing adequate screw position. 


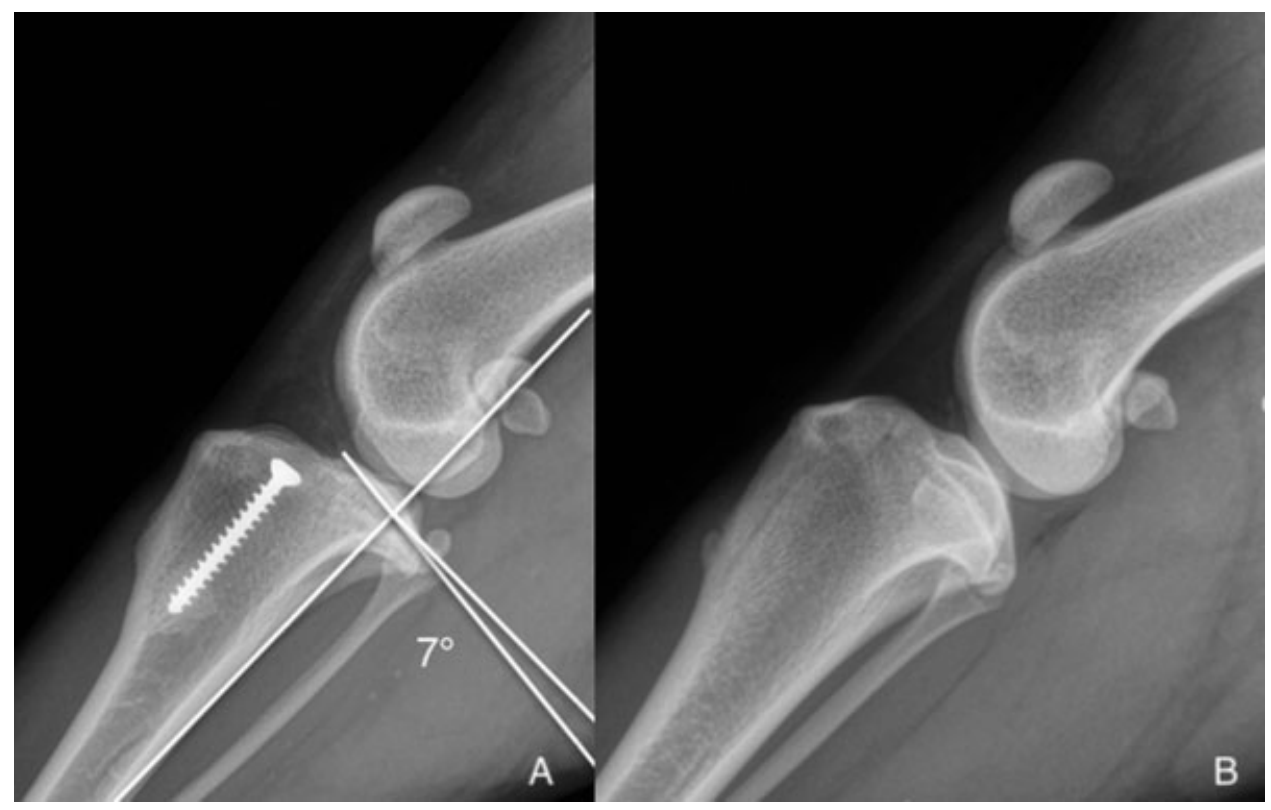

Fig. 3 Bilateral follow-up radiograph 8 months after surgery. (A) Operated side: No signs of osteoarthritis are visible. The final plateau angle is $7^{\circ}$ with caudal overhang of the proximal tibial epiphysis. (B) Contralateral side without overhang of the proximal tibial epiphysis.

Six-week postoperative follow-up revealed a dog free of lameness, pain-free mobilization of the stifle, normal range of motion and negative drawer signs. Healing of the tibial tuberosity avulsion fracture was progressing well on radiographs. Tibial plateau angle was $21^{\circ}$. The dog was re-evaluated 12 weeks after surgery. The tibial tuberosity growth plate was nearly closed on radiographs. Tibial plateau angle was $9^{\circ}$.

Last follow-up evaluation was performed 8 months postoperatively. The dog presented no lameness, and thighs' muscle masses were symmetrical. The operated stifle was stable and pain-free upon manipulation. Radiographs showed complete healing of the tibial tuberosity avulsion fracture and full closure of the tibial plateau growth plate. The screw head was covered by smooth, regular, new formed bone. The proximal tibia exhibited a tibial plateau caudally displaced, creating an overhang. There were no radiographical signs of osteoarthritis. Final tibial plateau angle was $7^{\circ}$ ( - Fig. 3). At telephone follow-up 18 months postoperatively, owners reported sustained and full functional recovery of the dog.

\section{Discussion}

This report illustrates the use of the proximal tibial epiphysiodesis as described by Vezzoni and colleagues ${ }^{14}$ for the successful treatment of a proximal tibial Salter-Harris type II fracture associated with avulsion of the tibial tuberosity in a 4.5-month-old Airedale Terrier. Reduction and stabilization of a proximal tibial physeal fracture can be challenging, and several ways of treatment have been proposed. $7,8,12,13,18$

Our elected method of treatment was based on the combination of several fracture features; that is, displacement of the fragments with a $40^{\circ}$ tibial plateau angle, undisrupted connection between tibial plateau and tuberosity, a growing patient, a 10-day period of time elapsed since the fracture occurrence, leading to a rather good stability of the fracture site. An attempt to reduce the tibial tuberosity would have disrupted soft tissues and early callus. Since the displacement was moderate, no functional handicap was expected. Non-surgical management is usually recommended as the initial treatment for dogs with minimally displaced tibial tuberosity avulsion fracture. ${ }^{19}$

The difference between pre- and immediate postoperative tibial plateau angles may be explained by the fact that although the screw was initially inserted in a neutral mode, it must have slightly displaced the tibial plateau in relation with the proximal tibial metaphysis.

Proximal tibial epiphysiodesis is a mini-invasive technique described by Vezzoni and colleagues ${ }^{14}$ to treat puppies affected by cranial cruciate ligament deficiency. The screw inserted in the centre of the cranial part of the tibial plateau arrests the cranial part of the tibial plateau growth. Consequently, the tibial plateau slope progressively decreases during the residual growth. Alternatively, proximal tibial epiphysiodesis by electrocauterization has been recently described. ${ }^{20,21}$ This procedure is thought to be less invasive than the screw technique, with a lower morbidity. It seems to provide the same final tibial plateau angle in a shorter time. ${ }^{21}$ However, we considered it non-applicable in our case as electrocauterizing the early callus could have affected the fracture healing process. Moreover, the screw contributed to the maintenance of reduction, and added stability.

We acknowledge the fact that using one single position screw to stabilize a type II Salter-Harris proximal tibial fracture might be seen as risky. When proposed by Vezzoni and colleagues, ${ }^{14}$ the epiphyseal screw had no role of stabilization and was only placed to prevent further growth of the cranial tibial plateau. We were not able to quantify, on one hand, the shear force that could be applied to the tibial 
plateau and, on the other, the shear strength of one $3.5 \mathrm{~mm}$ cancellous screw. Although guidelines for evaluating mechanical properties of implants are provided by the American Society for Testing and Materials, realistic clinical conditions evolving complex cortical geometry, cancellous architecture or bone properties cannot be fully duplicated. ${ }^{22}$ We nevertheless assumed that due to the 10-day period of time elapsed between the fracture and the surgical treatment, and given the very young age of the patient, some fibrous callus had already developed, hence some stability was already regained, mimicking more closely the use of one epiphysiodesis position screw, as previously described.

As mentioned by Vezzoni and colleagues, ${ }^{14}$ screw removal may be required in very young dogs. Occurrence of physis closure is in fact individual and breed related. The potential of remaining growth should be evaluated by looking at the physis width on pre- and post-op radiographs. ${ }^{14,15} \mathrm{~A}$ radiographical follow-up allows monitoring of the decreasing tibial slope. In case of overcorrection, the screw should be removed before the end of the growth phase. No screw removal was necessary in our case. Our final tibial plateau angle was $7^{\circ}$, an optimal result to prevent excessive strain on the cranial cruciate ligament, according to in vitro experimental testing. ${ }^{23}$

Radiographical follow-up is recommended for early diagnosis of angulation deformities secondary to epiphysiodesis screw insertion. Physeal closure can be symmetrical or asymmetrical. ${ }^{24}$ Angulation deformities have been found to be more frequent with a medial than with a lateral approach. ${ }^{14}$ Hence, our choice for a lateral approach with no final angulation observed. Screw position is essential, and the use of intraoperative fluoroscopy or radiographs is highly recommended. Despite a slight eccentricity of the screw observed on the postoperative radiograph, we could not detect any adverse consequence throughout the follow-up period.

No radiographical signs of osteoarthritis appeared during the growth of the dog. This highlights a major advantage of the mini-invasive procedure and suggests a stable knee joint and a cranial cruciate ligament integrity over time.

The noticeable increase in caudal overhang of the proximal tibial epiphysis is probably due to the proximal tibial plateau pivoting around the screw insertion point. This is also noticeable on the cases described by Vezzoni and colleagues, ${ }^{14}$ yet not reported previously.

To our knowledge, this is the first documented case of epiphysiodesis to treat a displaced proximal tibial physeal fracture in a growing dog. Thus, this is another indication of this technique, which, in this case, yielded excellent longterm outcome. As overcorrection could be a possible complication, radiographic follow-up is essential, allowing to decide whether and when screw removal may be required.

Based on this report, authors believe that epiphysiodesis has its place in the treatment arsenal of sub-acute, moderately displaced, Salter-Harris I or II proximal tibial fractures. Further cases are needed to demonstrate the efficiency of a single screw epiphysiodesis in the treatment of various types of proximal metaphyseal tibial fractures.

\section{Author Contribution}

Pierre P. Picavet, Martin Hamon and Marc Balligand contributed to the acquisition of data, data analysis and interpretation. Bernard Bouvy and Michael Lefebvre contributed to the conception of the study and study design, acquisition of data, and data analysis and interpretation. All authors drafted and revised and approved the submitted manuscript.

\section{Conflict of Interest}

None declared.

\section{References}

1 Boone EG, Johnson AL, Hohn RB. Distal tibial fractures in dogs and cats. J Am Vet Med Assoc 1986;188(01):36-40

2 Boone EG, Johnson AL, Montavon P, Hohn RB. Fractures of the tibial diaphysis in dogs and cats. J Am Vet Med Assoc 1986;188 (01):41-45

3 Unge r M, Montavon PM, Heim UFA. Classification of fractures of long bones in the dog and cat: introduction and clinical application. Vet Comp Orthop Traumato 1 1990;3:41-50

4 Seaman JA, Simpson AM. Tibial fractures. Clin Tech Small Anim Pract 2004;19(03):151-167

5 Schmökel H, Weber U, Hartmeier G. [Salter-II fracture of the proximal tibia with avulsion of the tuberositas tibiae in the dog]. Schweiz Arch Tierheilkd 1995;137(04):124-128

6 Skelly CM, McAllister H, Donnelly WJ. Avulsion of the tibial tuberosity in a litter of greyhound puppies. J Small Anim Pract 1997;38(10):445-449

7 Pratt JNJ. Avulsion of the tibial tuberosity with separation of the proximal tibial physis in seven dogs. Vet Rec 2001;149(12):352-356

8 Gower JA, Bound NJ, Moores AP. Tibial tuberosity avulsion fracture in dogs: a review of 59 dogs. J Small Anim Pract 2008;49(07): 340-343

9 Clements DN, Gemmill T, Corr SA, Bennett D, Carmichael S. Fracture of the proximal tibial epiphysis and tuberosity in 10 dogs. J Small Anim Pract 2003;44(08):355-358

10 Deahl L, Ben-Amotz R, Caceres AV, Agnello KA. Proximal tibial metaphyseal fractures in immature dogs. Vet Comp Orthop Traumatol 2017;30:237-242

11 Haynes KH, Biskup J, Freeman A, Conzemius MG. Effect of tibial plateau angle on cranial cruciate ligament strain: an ex vivo study in the dog. Vet Surg 2015;44(01):46-49

12 DeCamp CE, Johnston SA, Déjardin LM, Schaerfer SL. Fractures of the Tibia and Fibula. In: Brinker, Piermattei, and Flo's Handbook of Small Animal Orthopaedics and Fracture Repair. 5th ed. St. Louis, MO: Elsevier; 2016:670-707

13 von Pfeil DJF, Glassman M, Ropski M. Percutaneous tibial physeal fracture repair in small animals: technique and 17 cases. Vet Comp Orthop Traumatol 2017;30(04):279-287

14 Vezzoni A, Bohorquez Vanelli A, Modenato M, Dziezyc J, Devine Slocum T. Proximal tibial epiphysiodesis to reduce tibial plateau slope in young dogs with cranial cruciate ligament deficient stifle. Vet Comp Orthop Traumatol 2008;21(04):343-348

15 McBrien CS Jr, Vezzoni A, Conzemius MG. Growth dynamics of the canine proximal tibial physis. Vet Surg 2011;40(04):389-394

16 Ramirez J, Barthélémy N, Noël S, et al. Complications and outcome of a new modified Maquet technique for treatment of cranial cruciate ligament rupture in $82 \mathrm{dogs}$. Vet Comp Orthop Traumatol 2015;28(05):339-346

17 Slocum B, Slocum TD. Tibial plateau leveling osteotomy for repair of cranial cruciate ligament rupture in the canine. Vet Clin North Am Small Anim Pract 1993;23(04):777-795

18 Johnson MD, Lewis DD, Winter MD. Intraoperative use of a transarticular circular fixator construct to facilitate reduction 
and stabilisation of a proximal tibial physeal fracture in a dog. Aust Vet J 2017;95(05):161-166

19 von Pfeil DJF, Decamp CE, Ritter M, et al. Minimally displaced tibial tuberosity avulsion fracture in nine skeletally immature large breed dogs. Vet Comp Orthop Traumato 1 2012;25(06):524-531

20 Mesquita LR, Muzzi LAL, Giannico AT, et al. Proximal tibial epiphysiodesis in a growing dog. Acta Sci Vet 2016;44:113

21 Brienza PD, Muzzi LAL, Lacreta ACC Jr, Alves EGL, Guimaraes GC, Muzzi RAL. Electrocautery technique for proximal tibial epiphysiodesis in Labrador retriever puppies: experimental study. Arq Bras Med Vet Zootec 2016;68(06):1586-1594
22 Zdero R, Aziz M, Nicayenzi B. Pullout force testing of cortical and cancellous screws in whole bone. In: Zdero R, ed. Experimental Methods in Orthopaedic Biomechanics. Academic Press; 2016: 117-132

23 Warzee CC, Déjardin LM, Arnoczky SP, Perry RL. Effect of tibial plateau leveling on cranial and caudal tibial thrusts in canine cranial cruciate-deficient stifles: an in vitro experimental study. Vet Surg 2001;30(03):278-286

24 Demianiuk RM, Guiot LP. Reverse TPLO for asymmetrical -premature closure of the proximal tibial physis in a dog. J Small Anim Pract 2014;55(11):589-592 